\title{
Lusioersily
}

\section{Raman spectroscopic monitoring of the osteogenic differentiation of human mesenchymal stem cells}

McManus, L. L., Burke, GA., Boyd, A., Meenan, BJ., O'Hare, P., McCafferty, M., \& Modreanu, M. (2011). Raman spectroscopic monitoring of the osteogenic differentiation of human mesenchymal stem cells. The Analyst, 136, 2471-2481. https://doi.org/10.1039/c1an15167c

Link to publication record in Ulster University Research Portal

\section{Published in: \\ The Analyst}

Publication Status:

Published (in print/issue): 01/01/2011

DOI:

10.1039/c1an15167c

\section{Document Version}

Publisher's PDF, also known as Version of record

\section{General rights}

Copyright for the publications made accessible via Ulster University's Research Portal is retained by the author(s) and / or other copyright owners and it is a condition of accessing these publications that users recognise and abide by the legal requirements associated with these rights.

\section{Take down policy}

The Research Portal is Ulster University's institutional repository that provides access to Ulster's research outputs. Every effort has been made to ensure that content in the Research Portal does not infringe any person's rights, or applicable UK laws. If you discover content in the Research Portal that you believe breaches copyright or violates any law, please contact pure-support@ulster.ac.uk. 


\title{
Raman spectroscopic monitoring of the osteogenic differentiation of human mesenchymal stem cells
}

\author{
Lindsay L. McManus, ${ }^{\star a}$ George A. Burke, ${ }^{a}$ Mura M. McCafferty, ${ }^{a}$ Peter O’Hare, ${ }^{a}$ Mircea Modreanu, ${ }^{b}$ \\ Adrian R. Boyd ${ }^{a}$ and Brian J. Meenan ${ }^{a}$
}

Received 24th February 2011, Accepted 13th April 2011

DOI: 10.1039/c1an15167c

The differentiation of stem cells into multi-lineages is essential to aid the development of tissue engineered materials that replicate the functionality of their tissue of origin. For this study, Raman spectroscopy was used to monitor the formation of a bone-like apatite mineral during the differentiation of human mesenchymal stem cells (hMSCs) towards an osteogenic lineage. Raman spectroscopy observed dramatic changes in the region dominated by the stretching of phosphate groups (950-970 $\mathrm{cm}^{-1}$ ) during the period of 7-28 days. Changes were also seen at $1030 \mathrm{~cm}^{-1}$ and $1070 \mathrm{~cm}^{-1}$, which are associated with the $\mathrm{P}-\mathrm{O}$ symmetric stretch of $\mathrm{PO}_{4}{ }^{3-}$ and the $\mathrm{C}-\mathrm{O}$ vibration in the plane stretch of $\mathrm{CO}_{3}{ }^{2-}$. Multivariate factor analysis revealed the presence of various mineral species throughout the 28 day culture period. Bone mineral formation was observed first at day 14 and was identified as a crystalline, non-substituted apatite. During the later stages of culture, different mineral species were observed, namely an amorphous apatite and a carbonate, substituted apatite, all of which are known to be Raman markers for a bone-like material. Band area ratios revealed that both the carbonate-to-phosphate and mineral-to-matrix ratios increased with age. When taken together, these findings suggest that the osteogenic differentiation of hMSCs at early stages resembles endochondral ossification. Due to the various mineral species observed, namely a disordered amorphous apatite, a Btype carbonate-substituted apatite and a crystalline non-substituted hydroxyapatite, it is suggested that the bone-like mineral observed here can be compared to native bone. This work demonstrates the successful application of Raman spectroscopy combined with biological and multivariate analyses for monitoring the various mineral species, degree of mineralisation and the crystallinity of hMSCs as they differentiate into osteoblasts.

\section{Introduction}

The severity of disease and injury can impact upon the body's ability to self-heal resulting in the need for clinical intervention in order to maintain tissue function. One aim of regenerative medicine is to meet these needs with the production of functional tissue engineered bone material, which would be used in several applications where the loss of bone mass as a result of injury, disease or age presents a significant problem. ${ }^{1}$ Central to this is the re-establishment of tissue functionality by way of tissuespecific cells, such as human bone marrow derived stem cells. The success of a tissue engineered material is invariably dependent on

${ }^{a}$ Nanotechnology and Integrated Bioengineering Centre (NIBEC), University of Ulster, Shore Road, BT37 OQB, Northern Ireland. E-mail. charters-12@email.ulster.ac.uk; Fax: +44 (0)2890366863; Tel: +44(0) 2890368928

${ }^{b}$ Micro/Nanoelectronics Department, Tyndall National Institute, Lee Maltings, Cork, Ireland. E-mail: mircea.modreanu@tyndall.ie; Tel: +353 214904267 the ability of the cells to replicate the functionality of their tissue of origin.

It is often difficult to expand sufficient numbers of tissuespecific cells for use in regenerative medicine applications. Owing to their ability for self-renewal and multi-lineage differentiation, hMSCs have attracted widespread interest for their use in bone tissue engineering applications, and more specifically for their expansion and osteogenic differentiation potential in vitro. hMSCs have the ability to differentiate into a number of cells including osteoblasts, chondrocytes and adipocytes which then allow the formation of bone, cartilage and adipose tissue. ${ }^{2-6}$

With over two million replacement procedures carried out annually, bone is an ideal target for regenerative medicine therapies. Bone tissue is a complex material which is formed from an abundant intracellular matrix composed of organic proteins and inorganic mineral salts. It consists of a carbonated hydroxyapatite-like mineral supported on a hydrated, collagen-rich protein matrix. During fetal development of the mammalian skeletal system there are two essential processes resulting in the creation of new bone tissue, which are endochondral ossification and 
intramembranous ossification. Intramembranous ossification is the process by which flat bones such as those in the skull and collarbones are formed from connective tissue. Endochondral ossification occurs in the development of the long bones and most of the other bones in the body, and involves the mineralisation of cartilaginous template. ${ }^{7,8}$

Bone formation is both a complex and tightly regulated process that has been studied extensively. However, the composition of the mineral and matrix environments during the mineralisation process is not well understood. ${ }^{7,9}$ Researchers generally use gene expression, microarray analysis, protein expression and histological stains or any combination of these. ${ }^{7-11}$ However, these methods fail to identify the composition of the mineral and matrix environments formed during mineralisation, for example, alizarin red staining detects the presence of mineral and the initial deposition of mineral but is unable to characterise the mineral species. Raman spectroscopy overcomes this limitation by using information from vibrations of an ion or molecule within a mineral lattice, such as phosphate and carbonate, to identify the mineral formed during osteogenic differentiation, as well as the vibrations from the protein matrix. ${ }^{8}$ Indeed, the application of optical spectroscopy for stem cell differentiation has been extensively reviewed. ${ }^{12}$ To date, the study of the mineral and proteinaceous content in bone and also the non-collagenous proteins found in the extracellular matrix (ECM) has been dominated by the use of infrared spectroscopy $^{13-15}$ this is due to its sensitivity to the organic and inorganic elements of bone tissue. Recently, Krafft et al. carried out studies of hMSCs differentiating into osteoblasts, ${ }^{16}$ as have Salasznyk et al. ${ }^{17} \mathrm{Krafft}$ et al. used Fourier transform infrared spectroscopy in order to identify the differentiation state of individual hMSCs. Spectra from all samples highlighted bands which are characteristic of proteins, including amide III, II and I $\left(1250 \mathrm{~cm}^{-1}\right.$, $1540 \mathrm{~cm}^{-1}$ and $\left.1655 \mathrm{~cm}^{-1}\right), \mathrm{COO}^{-}\left(1400 \mathrm{~cm}^{-1}\right)$ and $\mathrm{CH}_{3}$ $\left(1455 \mathrm{~cm}^{-1}\right)$. Increases in the bands at 1025,1080 and $1152 \mathrm{~cm}^{-1}$ were also observed in the spectra of the control samples, these are suggested to be due to the accumulation of glycogen. In the spectra of the hMSCs that were cultured with osteogenic stimulants there was evidence of increased bands at 987, 1086 and $1112 \mathrm{~cm}^{-1}$, which are markers for a bone-like material, thus indicating the presence of a mineral species. ${ }^{16}$ Furthermore, various groups have highlighted the importance of phosphate symmetric stretching during cell differentiation using infrared spectroscopy. ${ }^{18-20}$

Despite this, the potential for in vivo applications is limited due to the strong absorption of water. Moreover the spatial resolution for infrared is restricted to approximately $10 \mu \mathrm{m}$ and therefore infrared spectroscopy is not the preferred technique for cell analysis. The low Raman scattering efficiency of water combined with the high sensitivity and spatial resolution, and its capability of obtaining spectra that are low in fluorescence using near-infrared lasers place the Raman technique as the favorable tool for cell analysis with a spatial resolution of between 0.1 and $1 \mu \mathrm{m}$. Also, the vibrational bands observed in Raman spectra allow discrimination between the chemical species that are present, and as such these bands are narrow so even small changes in both frequency and band shape can be observed.

Raman spectroscopy is a measurement of the wavelength and intensity of inelastically scattered light from a molecule and in biological tissues it is particularly sensitive to the vibrations of the mineralised species, such as the carbonate and phosphate bands found in bone tissue. This is due to the symmetrical vibrations of a molecule which are the most intense bands observed in Raman, and particularly in the Raman spectra of mineralised tissue, the most prominent vibrational band is the $\mathrm{PO}_{4}{ }^{3-} v_{1}$ symmetric stretch. Moreover, changes in both the frequency and shape of the PO symmetric stretch are dependent upon the mineral environment and will therefore change with mineral crystallinity and ionic incorporation., ${ }^{70,21}$ There are several bands to characterise the mineral and matrix in the Raman spectrum of bone which are summarised in Table $1 .^{7-9,22,23}$

Using a combination of chemical, molecular and biological characterisation techniques we investigated the bone nodules formed from hMSCs. This paper presents evidence of a carbonated apatite-like mineral formed in hMSCs at the earliest stages of formation and its development over 28 days. We are able to monitor changes in the $\mathrm{PO}_{4}{ }^{3-}$ symmetrical band shape and frequency, which reveal variations in crystallinity throughout the culture.

\section{Materials and methods}

\section{Cell culture}

hMSCs derived from the bone marrow of a 22 year old female (Lonza, UK) were used in this study. Cells were maintained in non-osteogenic media: DMEM high glucose supplemented with $10 \%$ foetal bovine serum (Invitrogen, UK). hMSCs were directed towards an osteogenic lineage by culturing hMSCs in non-osteogenic media supplemented with $10 \mathrm{nM}$ dexamethasone, $10 \mathrm{mM}$ $\beta$-glycerophosphate and $50 \mu \mathrm{M}$ ascorbic acid-2-phosphate (Sigma Aldrich, UK). hMSCs were seeded at a density of approximately $1 \times 10^{5}$ cells per $\mathrm{cm}^{2}$ onto $15 \mathrm{~mm} \times 15 \mathrm{~mm}$ fused silica substrates (The Technical Glass Company, UK). At 7 day intervals cells were fixed in $100 \%$ methanol for 10 minutes, samples were then air dried for 1 hour prior to Raman analysis.

\section{Alizarin red staining}

To confirm and quantify the extent of mineralisation, alizarin red staining (AR) was performed at days 7, 14, 21 and 28. Cells were washed three times in PBS, fixed and permeabilised in 4\% PFA for 20 minutes at room temperature then washed twice in deionised water to remove residual PFA. Cells were stained with $2 \%$ alizarin red for 30 minutes at room temperature with gentle

Table 1 Band assignments for Raman spectra of bone ${ }^{7-9,22,23}$

\begin{tabular}{|c|c|}
\hline $\begin{array}{l}\text { Raman } \\
\text { frequency shift } / \mathrm{cm}^{-1}\end{array}$ & $\begin{array}{l}\text { Peak } \\
\text { assignment (intensity) }\end{array}$ \\
\hline 855 & Hydroxyproline ring \\
\hline 876 & Hydroxyproline ring \\
\hline $950-964$ & $\mathrm{PO}_{4}{ }^{3-} v_{1}(\mathrm{P}-\mathrm{O}$ symmetric stretch $)$ \\
\hline $1001-1003$ & Phenylalanine \\
\hline 1030 & $\mathrm{PO}_{4}^{3-} v_{3}(\mathrm{P}-\mathrm{O}$ asymmetric stretch $)$ \\
\hline $1065-1070$ & $\mathrm{CO}_{3}^{2-} v_{1}(\mathrm{P}-\mathrm{O}$ symmetric stretch) \\
\hline 1245,1270 & Amide III \\
\hline 1445 & $\mathrm{CH}_{2}$ wag \\
\hline 1665 & Amide I \\
\hline
\end{tabular}


shaking. For the colorimetric assay 3.6\% (0.1 M) cetylpyridinium chloride was added to each sample for 45 minutes and the optical density was measured at $405 \mathrm{~nm}$.

\section{Raman spectroscopy}

Confocal Raman spectra of non-osteogenic and osteogenic cultured hMSC samples were obtained at seven day intervals up to 28 days using the Renishaw inVIA Reflex spectrometer connected to a Leica microscope and equipped with a $514 \mathrm{~nm}$ linefocus laser and a lateral spatial resolution of $0.1 \mu \mathrm{m}$. Before the acquisition of the Raman measurements the system was calibrated to the $520.5 \mathrm{~cm}^{-1}$ spectral line using an internal silicon reference. The laser beam was focused through a $50 \times(\mathrm{NA}=0.9)$ long working distance $(2 \mathrm{~mm})$ objective. Under these conditions, the laser spot size on the sample was $1.5 \mu \mathrm{m}$. Spectra covering the fingerprint region $\left(600-1800 \mathrm{~cm}^{-1}\right)$ were recorded at a resolution of $1.7 \mathrm{~cm}^{-1}$, with $200 \mathrm{~s}$ integration time per spectrum. Raman backscattered radiation was collected by the same objective passed through a $514 \mathrm{~nm}$ edge filter to block Rayleigh scattering and reflected laser light, before being directed through a $50 \mu \mathrm{m}$ slit into the spectrometer equipped with a 1800 lines/mm grating, and finally detected by a deep-depletion charge-coupled device detector. Eight Raman spectra were acquired for each seven day time point. The laser power $(<10 \mathrm{~mW})$ on the samples was carefully controlled in order to avoid the damage or burning of the samples.

\section{Data pre-processing}

It is accepted that spectral artifacts can have a considerable effect on the interpretation of data. Therefore it is necessary to distinguish between biochemical information and undesired effects. With Raman spectroscopy, is it known that before data interpretation can be performed, pre-processing of data should be carried out. To this end Origin 7 software (OriginLab, USA) was used to routinely pre-process the raw data. In the first instance, band alignment was performed to correct for instrumental spectral shifts. As such all spectra were normalised using the area of the $1449 \mathrm{~cm}^{-1}$ band. A multipoint baseline correction and smoothing by a 5-point Savitzky-Golay filter was also employed for each Raman spectrum. Contributions of the fused silica substrate were subtracted, however, small contributions may still be present. The area of individual vibrational bands was computed using the curve-fitting option of Origin 7 software.

\section{Univariate analysis}

The carbonate-to-phosphate ratios for each of the culture time points were calculated from an average of three carbonate/ phosphate band intensities. Three spectra were chosen because the intensity of the carbonate/phosphate band varied and there was less mineral present in some spectra, thus the spectra chosen had a strong mineral signal. The band intensity of the $\mathrm{CO}_{3}{ }^{2-}$ in plane vibrations at $1070 \mathrm{~cm}^{-1}$ was used as a measure of the carbonate content. The intensity of the baseline corrected $\mathrm{PO}_{4}{ }^{3-}$ phosphate symmetric stretch at $960 \mathrm{~cm}^{-1}$ was used as a metric of the phosphate content. A global average was obtained from the three averaged carbonate-to-phosphate ratios recorded for each culture time point.
The intensity of the $\mathrm{PO}_{4}{ }^{3-}$ phosphate symmetric stretch discussed previously was used as the metric of mineral content for the mineral-to-matrix ratio. The hydroxyproline peak at $\sim 850 \mathrm{~cm}^{-1}$ was used as a measure of the matrix content. It is noted that some researchers commonly use the amide I band envelope at $\sim 1600$ to $1720 \mathrm{~cm}^{-1}$ as a measure of the matrix content, however, this band can only be used when calculated from the area. This is due to the variations observed in the relative intensity of the $1665 \mathrm{~cm}^{-1}$ and $1685 \mathrm{~cm}^{-1}$ components with cross-linking. ${ }^{21,24}$ Phenylalanine was also considered but due to a contribution from non-collagenous matrix proteins it could not be relied upon here to provide robust data with respect to the matrix content. As with the carbonate-to-phosphate ratios, the mineral-to-matrix ratios were calculated for each of the culture time points from an average of three mineral/matrix band intensities and a global average was obtained..$^{7,8,10,21}$ Both ratios were calculated from the intensity of the bands chosen, because the intensity is less susceptible to background correction errors than the area.

\section{Multivariate analysis}

Principal component analysis (PCA) is a multivariate analysis technique that is widely used to simplify a complex dataset of multiple dimensions. It allows the reduction of a set of variables into a smaller set of principal components that capture the maximum variance in the data. This statistical method of analysis was preferred because it is able to resolve spectral components that are present in different proportions. This is extremely important when bone is the material being analysed, as it is heterogeneous in nature, which results in considerable variations in its spectra. The order of the principal components represents their importance to the dataset, so the greatest variance is illustrated by the first principal component (PC1), the second greatest variance is the second principal component (PC2) and so forth. ${ }^{9,25,26}$ A scatter plot is generated from the data may show groups of points representing variations within the dataset.

\section{Immunocytochemistry}

After 14 and 28 days, cells were washed in large volumes of icecold PBS $(3 \times 5 \mathrm{~min})$ and fixed and permeabilised in $4 \%$ paraformaldehyde containing $0.1 \%$ Triton X-100 for 20 minutes at 4 ${ }^{\circ} \mathrm{C}$ and stained with bone specific proteins, including type-II collagen (Sigma, UK), osteocalcin, osteopontin, alkaline phosphatase and SPARC (Santa Cruz Biotechnology Inc., USA). Following washing, samples were treated with a blocking solution (PBS containing 1.0\% BSA, 5\% normal goat serum) for 30 minutes at room temperature. After blocking, the samples were washed in PBS and then incubated with the appropriate primary antibodies for 1 hour before exposure to the opposite secondary antibodies. Following secondary antibody incubation, cells were counterstained with DAPI nucleic acid stain (Molecular Probes, Inc. Invitrogen). After washing twice in PBS, substrates were mounted in Vectorshield mounting media (Vector Laboratories, UK). The substrates were sealed using a clear nail varnish and examined using a Carl Zeiss LSM5 Pascal (Carl Zeiss MicroImaging, Germany) Confocal Laser Scanning Microscope (CLSM). The diluent for all antibodies was PBS-1\% BSA. 


\section{Real time-polymerase chain reaction (RT-PCR)}

In this study, the hMSC cells were cultured as outlined previously and the mRNA expression of collagen type-II was investigated at days 7, 14, 21 and 28 of culture. RNA was isolated using Tripure reagent (Roche Diagnostics, UK) according to the manufacturer's instructions. Briefly, cells were lysed for 5 minutes at room temperature. Chloroform was then added to the cell lysate and incubated for 15 minutes. For phase separation the sample was centrifuged at $6000 \mathrm{~g}$ for 15 minutes at $4{ }^{\circ} \mathrm{C}$. For RNA isolation, RNA was precipitated by adding isopropanol, samples were mixed by inversion several times and incubated at room temperature for 10 minutes, samples were then centrifuged at $6000 \mathrm{~g}$ for 15 minutes at $4{ }^{\circ} \mathrm{C}$. The RNA pellet was washed with $75 \%$ ethanol and the sample was centrifuged at $6000 \mathrm{~g}$ for 10 minutes. Following centrifugation and removal of ethanol the RNA pellet was air-dried for 5-10 minutes and the pellet resuspended in RNase free molecular biology grade water (SigmaAldrich, UK). RNA was quantified by measuring the absorbance at $260 \mathrm{~nm}$. The concentration of the RNA was evaluated by A260/A280 ratio (at least 1.9 taken to be pure) using an Implen Nanospectrophotometer (Implen, Germany).

Following RNA extraction, first strand complementary DNA (cDNA) was synthesised using the transcriptor first strand cDNA synthesis kit (Roche Diagnostics, UK) with random hexamer primers according to the manufacturer's protocol. Briefly, the samples were incubated for 10 minutes at $25^{\circ} \mathrm{C}$, followed by 60 minutes at $50{ }^{\circ} \mathrm{C}$. To inactivate reverse transcriptase activity the samples were heated to $85^{\circ} \mathrm{C}$ for 5 minutes. The cDNA reaction was performed in Thermo OCR Sprint (Thermo Electron Corporation, UK).

Quantitative real time PCR was carried out using a Roche Light-Cycler 2.0 (Roche, Mannheim, Germany), using the following primers: collagen-II $\alpha$ (Col2A1) forward, 5'-TGGTGCTAATGGCGAGAAG-3' and reverse, 5'-CCCAGTCTCTCCACGTTCAC-3' (accession number: NM001844.4) and GAPDH forward, 5'-AGCCACATCGCTCAGACAC-3' and reverse 5'-GCCCAATACGACCAAATCC-3' (accession number NM-002046.3). hMSC cDNA was denatured at $95{ }^{\circ} \mathrm{C}$ for $10 \mathrm{~min}$, and 45 cycles of three-step amplification (denaturation, $95{ }^{\circ} \mathrm{C}$ for $10 \mathrm{~s}$; annealing, $60{ }^{\circ} \mathrm{C}$ for $15 \mathrm{~s}$; and, elongation $72{ }^{\circ} \mathrm{C}$ for $10 \mathrm{~s}$ ). At the end of the cycling, subsequent melt curve analysis was performed between 60 and $97{ }^{\circ} \mathrm{C}$ with a heating rate of $0.1{ }^{\circ} \mathrm{C} \mathrm{s}^{-1}$ in continuous acquisition mode to check the specificity of amplication and the possible presence of byproducts. All samples were amplified in triplicate, and the GAPDH gene was used as the reference to normalise the data using the Roche software.

\section{Results and discussion}

\section{Cell morphology}

Both non-osteogenic and osteogenic cell cultures underwent differing growth patterns, as expected. As can be seen in Fig. 1, for cells grown in non-osteogenic media, there was no evidence of mineral formation. Non-osteogenic hMSCs maintained a fibroblast-like morphology with a spindle-like shaped cell body throughout the 28 day culture period. The hMSCs grown in osteogenic media showed evidence of an opaque white growth on

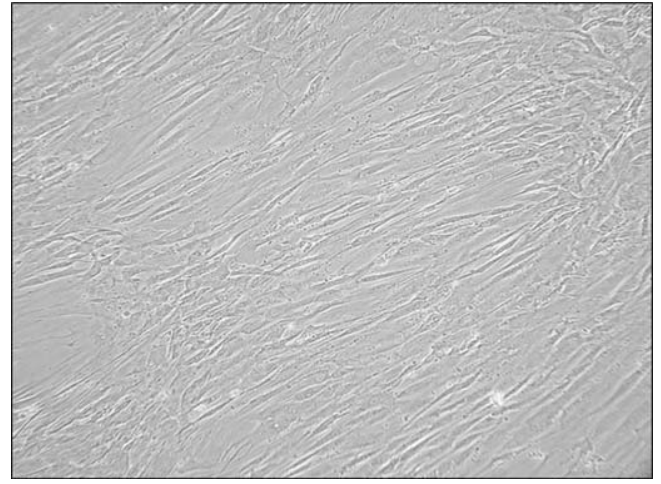

Fig. 1 Phase contrast microscope image of hMSCs cultured for 28 days in non-osteogenic media. Magnification $100 \times$.

the fused silica surface, which increased in both density and thickness over the culture period, however, mineralisation was not apparent during the first seven days of culture (as shown in Fig. 2A). From as early as day 7, hMSCs cultured in osteogenic media exhibited a different morphology. At this point the hMSCs lost their fibroblast appearance and became cuboidal in shape (as shown in Fig. 2A). By day 14 there was evidence of discrete patches of cellular/protein aggregation, and from the literature it is known that such aggregates occur prior to nodule formation and mineralisation (as shown in Fig. 2B). hMSCs cultured in osteogenic media for 21 and 28 days showed the formation of dense mineralised structures, which are described as "bone nodules" (as shown in Fig. 2C and D). ${ }^{7,8,21}$ At day 28 there is also evidence to suggest that there are what is presumed to be migratory osteoblasts present at the outer regions of the cell monolayer and it is envisaged that if samples were to remain in culture in the presence of osteogenic media that new bone material would be laid down by these cells, although further work is required for confirmation. Similarly to Gentleman et al., the nodules formed from hMSCs occurred in discrete patches throughout the culture and cellular aggregation was observed preceding mineralisation. ${ }^{7}$ Despite this, AR staining of osteogenic hMSCs revealed positive staining for calcium deposition which significantly increased through each 7 day interval (as shown in Fig. 3A-D). Quantification of AR staining of hMSCs
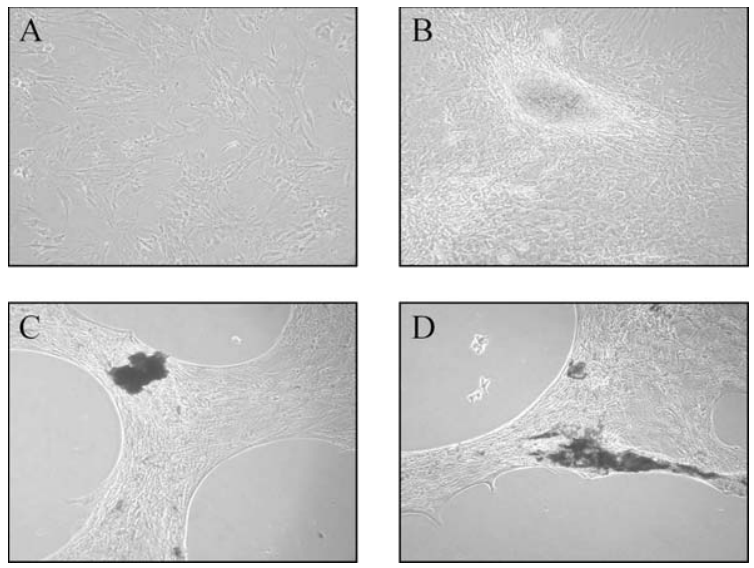

Fig. 2 Phase contrast images of hMSCs cultured in osteogenic media for 7 days (A), 14 days (B), 21 days (C) and 28 days (D). Magnification 100×. 

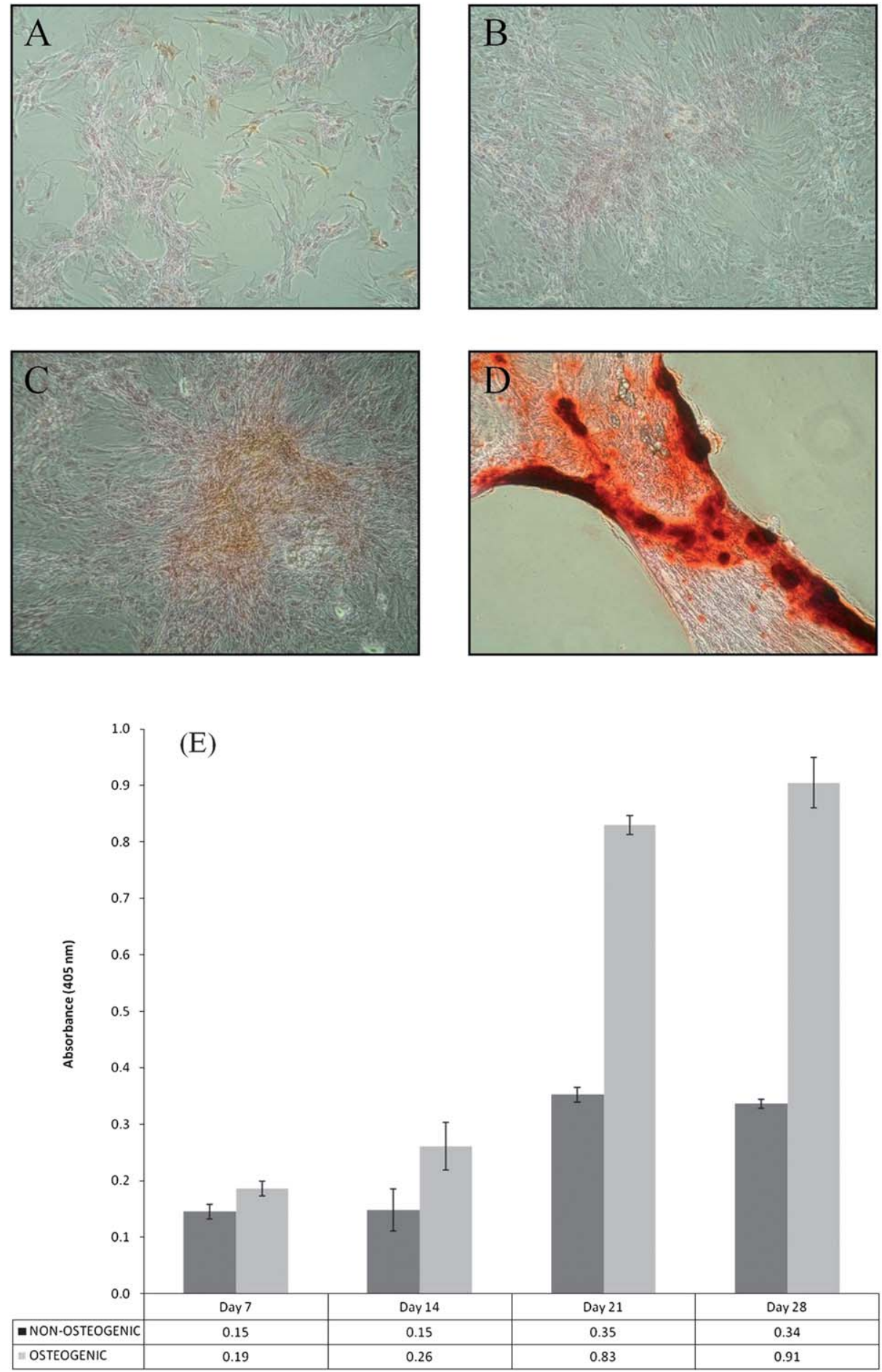

Fig. 3 Alizarin red staining of hMSCs cultured in osteogenic media for 7 days (A), 14 days (B), 21 days (C) and 28 days (D). Magnification 100×. (E) Quantification of Alizarin red staining of hMSCs cultured in non-osteogenic and osteogenic media.

cultured in non-osteogenic media remains constant from days $0-14$, with an increase observed between days 14-21, which is thought to be due to cell proliferation. The AR staining of hMSCs cultured in osteogenic media increases with culture time, with staining increasing rapidly between days 14-21 with day 28 showing the maximum amount of AR staining (as shown in Fig. 3E). Osteoblasts initiate the calcification process and produce what is referred to as osteoid. Osteoid is an intracellular matrix composed of collagen fibrils and fibres combined with a proteinaceous component. During the process of ossification, hardening and calcification of the matrix occurs around the osteoid. ${ }^{27-29}$ It is therefore suggested that the significant increase of AR staining between days 14-21 is due to mineralisation and calcification of the osteoid laid down by osteoblasts.

The standard biological techniques used to assess morphology and histology provide information that is limited because it 
cannot distinguish between nodules, therefore a different approach was required.

\section{Premineralised hMSCs}

The Raman spectra of both non-osteogenic hMSCs and hMSCs grown in osteogenic media for 7 days (as shown in Fig. 4 and 5A, respectively) are dominated with protein features, including the amide I band $\left(1659 \mathrm{~cm}^{-1}\right)$, skeletal $\mathrm{C}-\mathrm{C}$ vibrations $\left(936 \mathrm{~cm}^{-1}\right)$ and amide III vibrations $\left(1284 \mathrm{~cm}^{-1}\right)$. Peaks indicative of lipids at $786,1095,1575 \mathrm{~cm}^{-1}$ and phospholipids at $1065,1127,1301 \mathrm{~cm}^{-1}$ are observed. $\mathrm{C}-\mathrm{N}-\mathrm{H}$ amide III stretches $\left(1246\right.$ and $\left.1269 \mathrm{~cm}^{-1}\right)$, hydroxyproline $\left(950 \mathrm{~cm}^{-1}\right)$ and proline $\left(921 \mathrm{~cm}^{-1}\right)$ are also evident. The Raman spectrum of hMSCs grown in non-osteogenic media show no evidence of a $\mathrm{PO}_{4}{ }^{3-}$ symmetric stretching band indicating that although the morphological characteristics of the cells changed as they became confluent there were no significant changes observed spectrally.

At day 14 there were no nodules formed in the culture so the protein/cell aggregates described previously were examined. The Raman spectrum of hMSCs cultured for 14 days in osteogenic media shows changes in the intensity of protein bands, however, the lipid and phospholipid peaks show no distinguishable differences (as shown in Fig. 5B). The regions in the spectra that correspond to the proteinaceous matrix of the cell aggregates reveal a Raman spectrum that can be associated with that of collagen type-II. A collagen type-II template is produced prior to bone mineralisation and is a known to be the main component of cartilage which is produced during endochondral ossification. Similar findings were observed by Gentleman et al. who after the analysing the covariance matrix revealed a correlation coefficient of 0.96 when comparing animal derived MSCs with type-II collagen. ${ }^{7}$ In order to further confirm that collagen type-II was present, immunocytochemical localisation with antibodies to type-II collagen was performed on hMSCs cultured in osteogenic media for 14 days (as shown in Fig. 5C and D). Significant positive staining of collagen type-II was observed in the hMSC cell/protein aggregates present at day 14. Real-time PCR was then performed to investigate the expression of the collagen typeII gene through the 28 day culture and melt curve analysis was

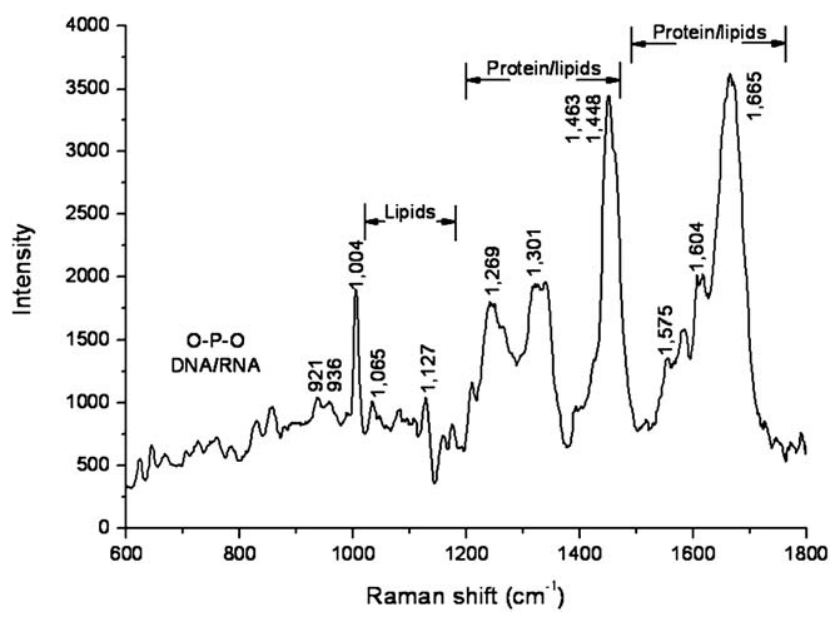

Fig. 4 Raman spectra of hMSCs cultured in non-osteogenic media for 28 days.
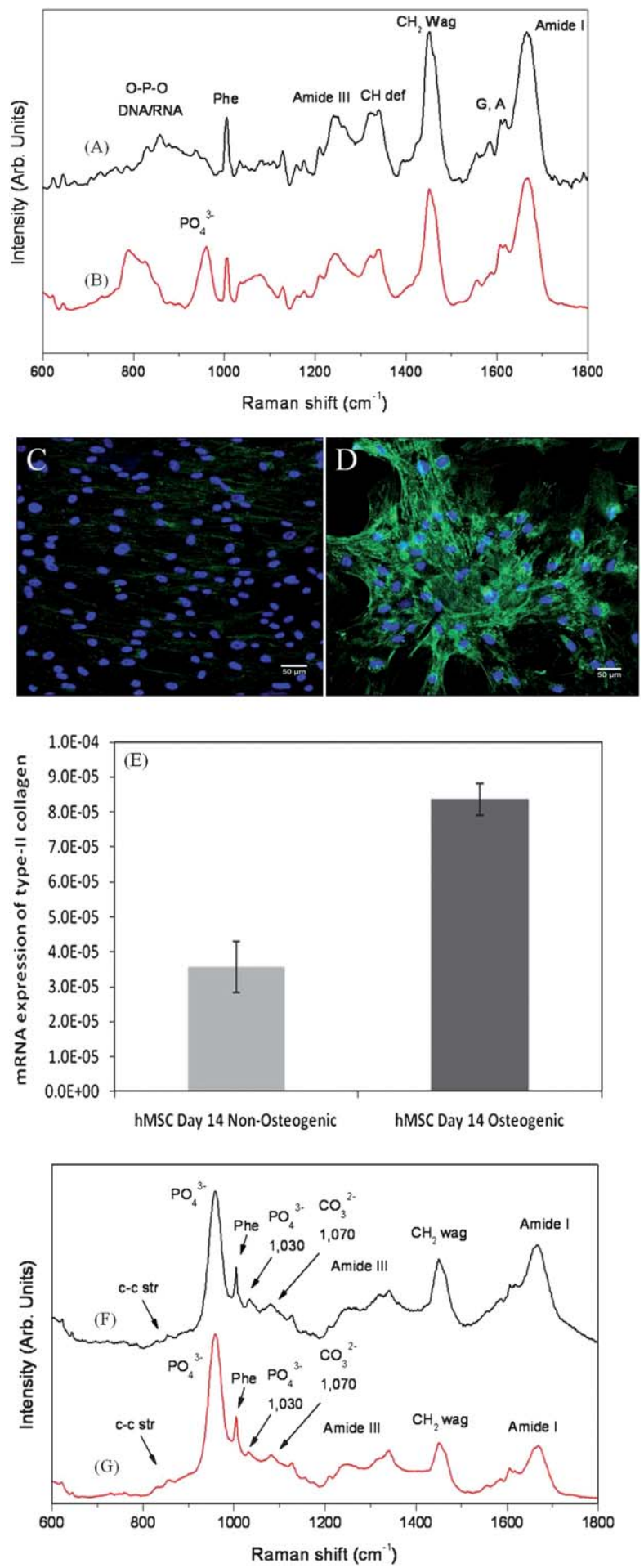

Fig. 5 Raman spectra of premineralised hMSCs cultured in osteogenic media at day 7 (A), day 14 (B), day 21 (F), day 28 (G). Immunocytochemical localisation of type-II collagen in hMSCs cultured for 14 days in (C) non-osteogenic media and (D) osteogenic media. (E) Relative expression of hMSCs cultured in non-osteogenic media compared with the expression of hMSCs cultured in osteogenic media for 14 days determined by quantitative real-time reverse-transcriptase polymerase chain reaction (RT-PCR) of the type-II collagen (Col2A1) gene. Note that the $y$ axis is a logarithmic scale. 

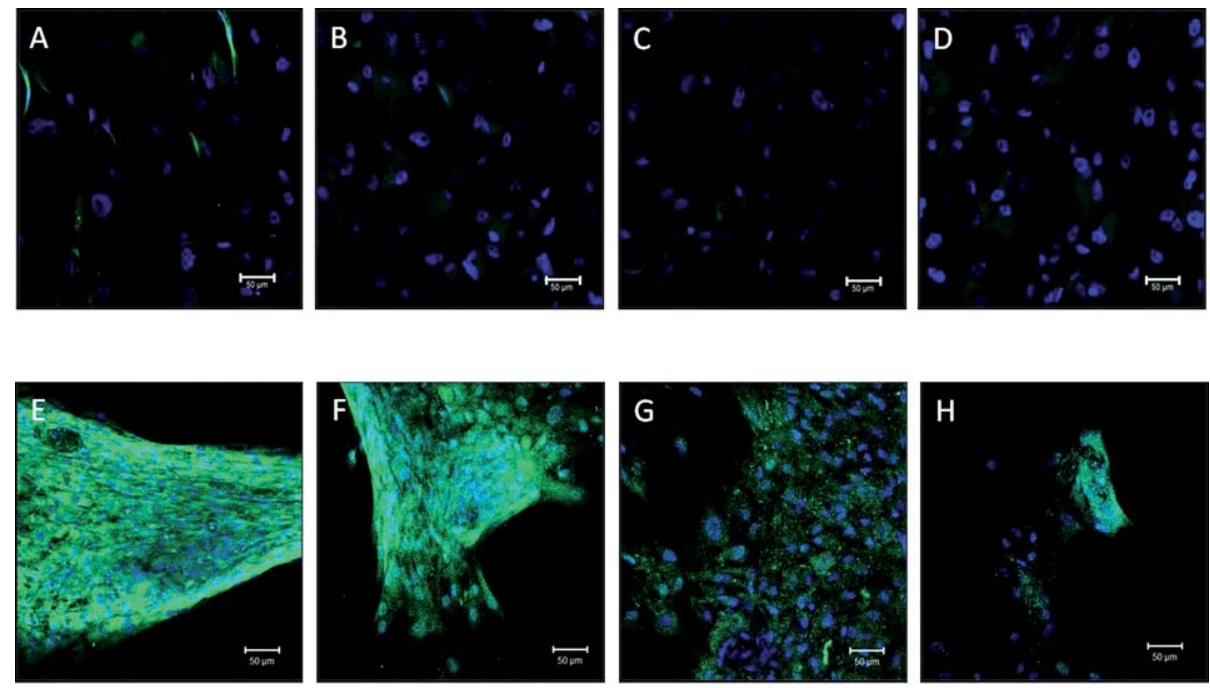

Fig. 6 Immunocytochemical localisation of bone specific proteins SPARC, osteocalcin, osteopontin and alkaline phosphatase of hMSCs culture in non-osteogenic media (A-D) and osteogenic media (E-H) for 28 days.

carried out to check the specificity of amplication and the possible presence of byproducts (not shown here). The gene expression for collagen type-II indicates a significant up-regulation at day 14 when compared to the day 14 control, as illustrated in Fig. 5E. The Raman spectrum of hMSCs cultured for 14 days in osteogenic media shows the inclusion of a $\mathrm{PO}_{4}{ }^{3-}$ symmetric stretch at approximately $960 \mathrm{~cm}^{-1}$ in the spectra, indicating that although no nodule formation was observed, the cell/protein aggregates reveal the beginning of mineralisation. This therefore highlights that the cells have differentiated into osteoblasts, which have begun to calcify. ${ }^{7}$

\section{Mineralised hMSCs}

By days 21 and 28, hMSCs cultured in osteogenic media contained mineralised nodules and the peaks indicative of collagen type-II were no longer detected in the Raman spectra. For these samples instead the Raman spectrum revealed bands that are characteristic of bone tissue and is dominated by the $\mathrm{PO}_{4}{ }^{3-}$ symmetric stretch near $960 \mathrm{~cm}^{-1}$ which is associated with hydroxyapatite $\mathrm{Ca}_{10}\left(\mathrm{PO}_{4}\right)_{6}(\mathrm{OH})_{2}$. Weaker peaks are also observed at carbonate (substituted $\mathrm{CO}_{3}{ }^{2-}$ in plane vibrations $1070 \mathrm{~cm}^{-1}$ ) and phosphate $\left(1030-1100 \mathrm{~cm}^{-1}\right)$ modes (as shown in Fig. $5 \mathrm{~F}$ and $\mathrm{G})$. The de novo bone mineral is deposited on an ECM of which the main composition is collagen type-I. This can be observed in the Raman spectra by the inclusion of prominent protein bands including amide I $\left(1665 \mathrm{~cm}^{-1}\right)$, amide III (1245$1270 \mathrm{~cm}^{-1}$ ) and a $\mathrm{CH}_{2}$ wag $\left(1445 \mathrm{~cm}^{-1}\right){ }^{7,10,11,21,30,31}$ Immunocytochemistry was performed to confirm the presence of bone specific proteins, SPARC, osteocalcin, osteopontin, and alkaline phosphatase of hMSCs cultured for 28 days in both osteogenic and non-osteogenic media. Significant positive staining on osteogenic cultures is observed when compared to the nonosteogenic control taken at the same time-point, as shown in Fig. 6A-H.

The Raman spectra of the mineralised nodules observed at days 21 and 28 have similarities, however, changes in the mineral environment can be characterised by variations in the phosphate band shape and frequency. Therefore univariate peak analysis was carried out to aid in the understanding of bone formation at different stages of development, as detailed in Table 2. ${ }^{7,9,24}$

The carbonate-to-phosphate ratios and mineral-to-matrix ratios for each of the culture time points were calculated from an average of three band intensities (as shown in Fig. 7A and B).$^{7-10,21,24}$ The carbonate-to-phosphate ratio is a measure of the inclusion of carbonate into the mineral lattice. Fig. 7A shows the carbonate-to-phosphate ratios from hMSCs cultured in osteogenic media for up to 28 days. The carbonate-to-phosphate ratio for day 7 is not shown because the carbonate band intensity was too weak to allow accurate calculations to be performed. The other culture time points revealed an increase in the carbonateto-phosphate ratio and this occurred throughout the culture. It was observed that the carbonate-to-phosphate ratio was significantly higher in hMSCs cultured for 21 days when compared to those cultured for 14 days, suggesting that carbonate substitution occurred most rapidly between days 14 and 21 . At day 28 the carbonate-to-phosphate ratio shows an increase from 0.36 to 0.37 indicating that carbonate substitution was significantly reduced after 21 days in culture. The mineral-to-matrix ratio of hMSCs cultured for 28 days was significantly higher between all culture time points and this increased with culture time, with the largest increase occurring between days 21 and 28. This suggested that an increased amount of mineral is found in older tissue (as shown in Fig. 7B). Mineral deposition occurs most rapidly during days 14 and 28 and as a consequence during this time the mineral-to-matrix ratio increased quite dramatically. Similarly, the extent of mineral crystallinity can be calculated from the FWHM of the $\mathrm{PO}_{4}{ }^{3-}$ band. Results indicate that the crystallinity of hMSCs cultured for 21 days in osteogenic media is significantly higher than the crystallinity observed for those at day 28 , which is illustrated by a lower FWHM., ${ }^{7,9,24}$ When all univariate peak analysis are taken together, this data suggests that although the hMSCs formed dense mineral at day 28, this mineral was of a lower crystallinity and had a higher degree of carbonate substitution than at day 21 . 
Table 2 Table detailing the phosphate peak $\left(\mathrm{PO}_{4}{ }^{3-}\right)$ parameters and mineral properties as determined by univariate analysis carried out by curve fitting Raman spectra of hMSCs cultured for 7-28 days. $n$ Represents the number of spectra used for each group

\begin{tabular}{lllll}
\hline & \multicolumn{2}{l}{ Phosphate peak } & & \\
\cline { 2 - 5 } & Frequency/cm ${ }^{-1}$ & FWHM/cm & \\
& - & - & Mineral-to-matrix ratio & Carbonate-to-phosphate ratio \\
\hline Day 7 $(n=8)$ & $960.72 \pm 1.96$ & $75.04 \pm 39.51$ & $0.38 \pm 0.52$ & $-7.47 \pm 4.17$ \\
Day 14 $(n=8)$ & $958.84 \pm 0.93$ & $72.19 \pm 4.86$ & $19.14 \pm 1.42$ & $0.19 \pm 0.20$ \\
Day 21 $(n=8)$ & $960.2 \pm 1.43$ & $92.2 \pm 8.09$ & $35.16 \pm 5.95$ & $0.36 \pm 0.86$ \\
Day 28 $(n=8)$ & & & $0.37 \pm 0.17$ \\
\hline
\end{tabular}

Univariate analysis enabled differences in crystallinity to be monitored. This was determined by resolving changes of the phosphate band shape and frequency, however, such analysis is unable to characterise the various mineral environments that are present in bone. Therefore, multivariate techniques including PCA and factor analysis were performed on the Raman spectra of day 28 hMSCs cultured in osteogenic media. The 2D plot comparing the four different culture time points for the osteogenic hMSCs is displayed in Fig. 8A. By focusing first on the
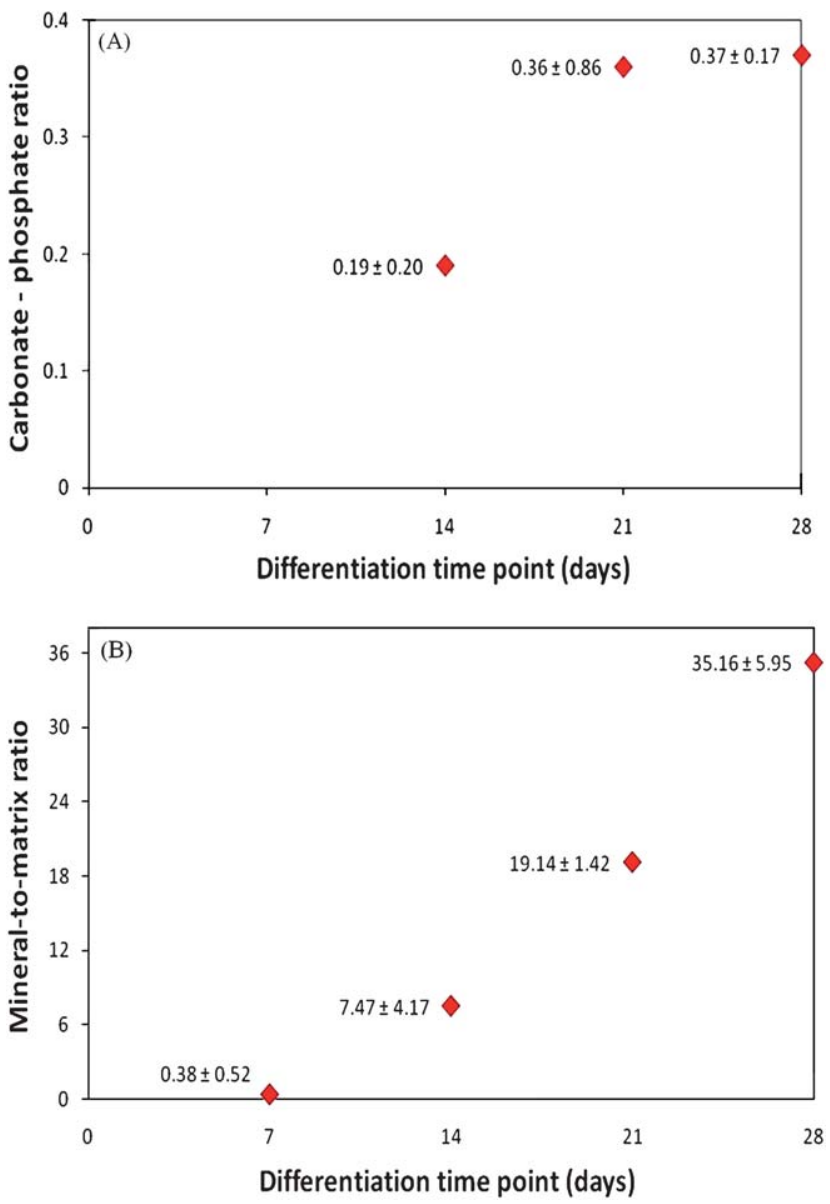

Fig. 7 (A) Carbonate-to-phosphate ratio $\left(\mathrm{CO}_{3}{ }^{2-}, 1070 \mathrm{~cm}^{-1} ; \mathrm{PO}_{4}{ }^{3-}, 960\right.$ $\mathrm{cm}^{-1}$ ) from hMSCs cultured in osteogenic media for 28 days. Day 7 is not shown because the carbonate band intensity is too weak to allow an accurate calculation. (B) Mineral-to-matrix ratio $\left(\mathrm{PO}_{4}^{3-}, 960 \mathrm{~cm}^{-1}\right.$; Hydroxyproline $850 \mathrm{~cm}^{-1}$ ) from hMSCs cultured in osteogenic media for 28 days. The values shown are averaged carbonate-to-phosphate ratios and mineral-to-matrix ratios for each time point. difference between the four culture times, a clear discrimination can be seen between all time points as expected from the differences seen in their Raman spectra. PC1 and PC2 show a clear difference between the hMSCs at days 21 and day 28 which are clustered in quite compact groups while the score plot showed that the hMSCs at days 7 and 14 are considerably more scattered. PC1 and PC2 account for 54\% and 30\% of the variance of data, respectively, however, $\mathrm{PC} 2$ is the distinguishing $\mathrm{PC}$ that is responsible for group separation. While there is distinct clustering of the four time points, overlap is still present which is presumably due to their many functional aspects. It also highlights that there are chemical and molecular differences between the culture times. Overall, PCA shows good separation in the

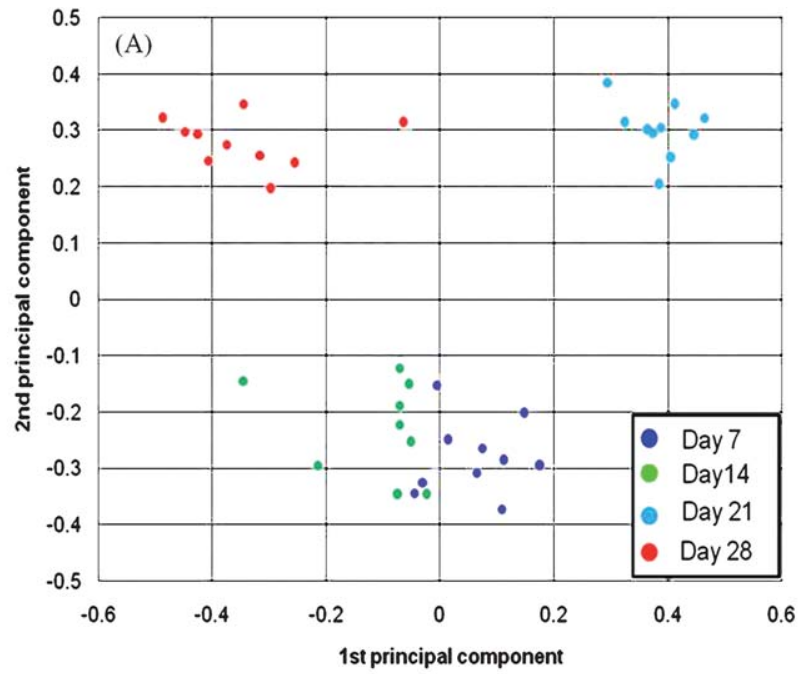

(B)
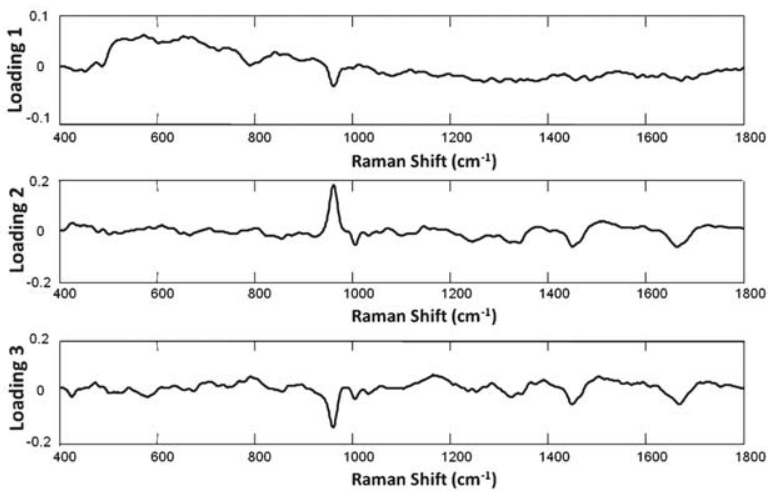

Fig. 8 (A) 2D scatter plot using scores of PC1 and PC2 of Raman spectra of hMSCs cultured in osteogenic media for 7-28 days and (B) plot of the loadings corresponding to the first three principal components. 

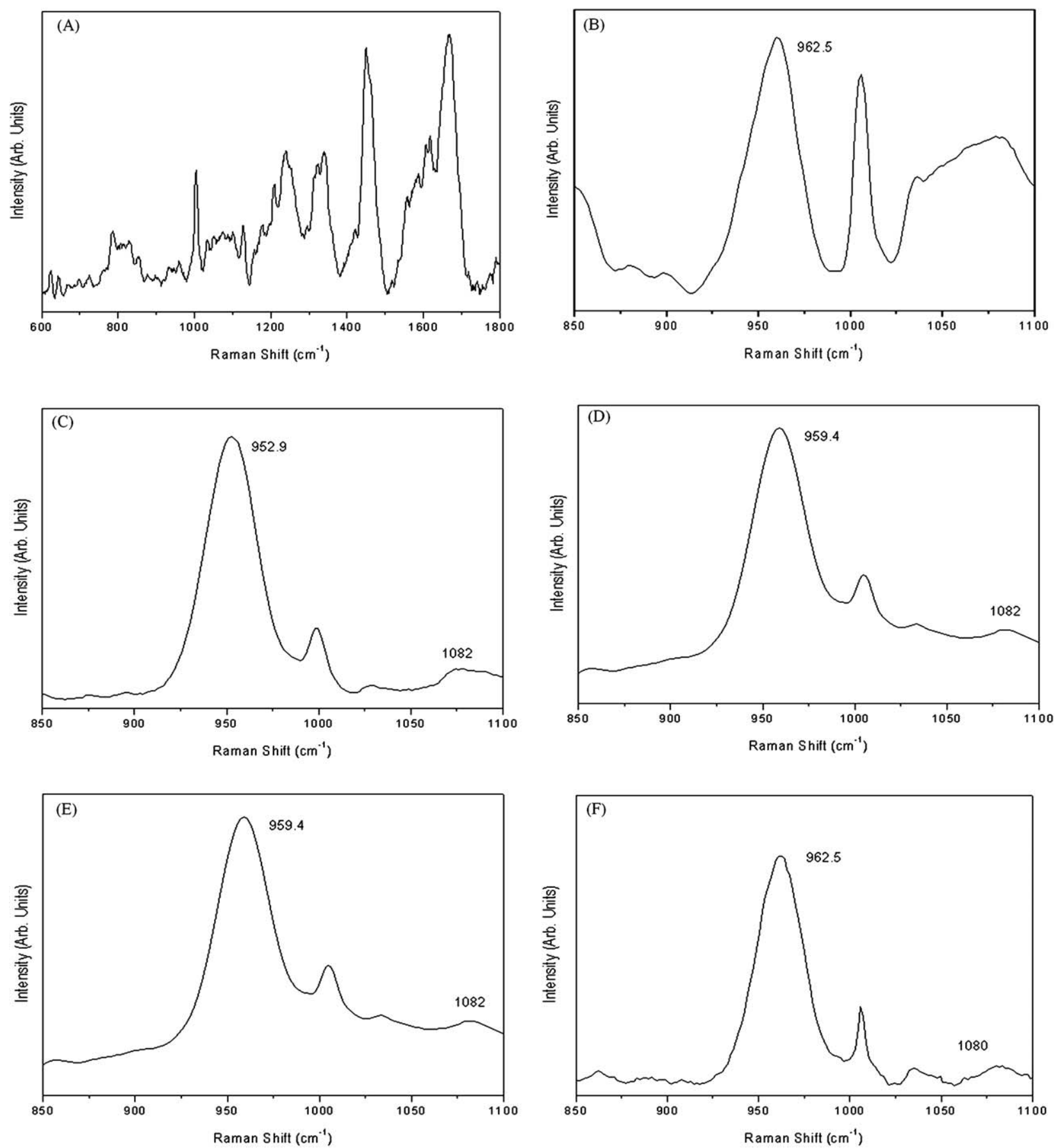

Fig. 9 Factor analysis of mineralised nodules after 14, 21 and 28 days in culture. (A) Matrix factor describing the protein component of mineralised nodules of hMSCs. Mineral factors produced for day 14 (B), day 21 (C and D) and day 28 (E and F).

Raman spectra of hMSCs cultured for 21 and 28 days but no major separation in the Raman spectra at days 7 and 14 is observed. The corresponding loading plots, shown in Fig. 8B, highlight the possible changes which fall around $500-1000 \mathrm{~cm}^{-1}$, and more specifically $\sim 960 \mathrm{~cm}^{-1}$. With regards to the spectra of mineralised nodules, the principal components can be added and subtracted from one another (rotated) resulting in 'factors' that represent the various mineral and matrix components that are present in a sample. As mentioned previously, both the position and shape of the $\mathrm{PO}_{4}{ }^{3-}$ band highlight changes in mineral crystallinity, therefore it is especially important when factor analysis is used to reveal how various tissue components change during the culture. ${ }^{7,9}$

Generally the apatite environments of mineralised tissue can be classified into three broad mineral categories which contain three different $\mathrm{PO}_{4}{ }^{3-}$ band frequencies. A disordered amorphous apatite lattice is represented by a band with a Raman shifted frequency between 945 and $950 \mathrm{~cm}^{-1}$. It is not known if this band frequency originates from an amorphous calcium phosphate or from an A-type carbonate substitution where carbonate is 
substituted for hydroxide; therefore, the term disordered phosphate is used to describe this mineral species. A B-type carbonate-substituted apatite produces a Raman frequency between the region of 955 and $959 \mathrm{~cm}^{-1}$. Finally, in a crystalline non-substituted hydroxyapatite, the $\mathrm{PO}_{4}{ }^{3-}$ band frequency is between 962 and $964 \mathrm{~cm}^{-1} .9,21,32-34$ Bone tissue is a complex material that is composed of various amounts of each of these three mineral species, and consequently, the resulting $\mathrm{PO}_{4}{ }^{3-}$ band is a sum of all three bands. The most commonly identified mineral species in the spectrum of bone tissue is a carbonated apatite phosphate. ${ }^{9}$ Hence, in a Raman spectrum the frequency and position of the phosphate band is in the $955-959 \mathrm{~cm}^{-1}$ region. This band is symmetric due to the inclusion of varying amounts of disordered phosphate and the more crystalline nonsubstituted phosphate to the left and right sides of the main band. ${ }^{7,9}$ Factor analysis of differentiated hMSC produced one mineral factor for day 14, and two mineral factors for both days 21 and 28, respectively, (as shown in Fig. 9A-F). At day 21 the two dominant mineral species factors indicate an amorphous phosphate species (as shown in Fig. 9C) and a B-type carbonatesubstituted apatite (as shown in Fig. 9D). Similarly factors of day 28 hMSCs yielded a B-type carbonate-substituted apatite (as shown in Fig. 9E) and a crystalline non-substituted hydroxyapatite, as shown in Fig. 9F. In contrast, factor analysis of day 14 produced only one mineral factor: a crystalline nonsubstituted hydroxyapatite (Fig. 9B). Gentleman et al. indicated that the Raman signal of synthetic carbonated hydroxyapatite is similar to that observed of a crystalline non-substituted apatite which was observed at day $14 .^{7}$ This data indicates that the hMSCs nodules examined at days 21 and 28 contained mineral species that are similar to what is expected in native bone, namely a disordered amorphous apatite, a B-type carbonate-substituted apatite and a crystalline non-substituted hydroxyapatite. In contrast, day 14 was dominated by one mineral factor which can be compared to synthetic hydroxyapatite. This time point failed to exhibit the more complex interaction of mineral species observed at days 21 and 28.,7,11,30,31

\section{Conclusion}

These findings suggest that during the early stages of the osteogenic differentiation of hMSCs, a collagen type-II template is produced before mineralisation occurs, therefore this reflects the process of endochondral ossification that was described previously. Briefly, this process is initiated by the hMSCs which aggregate in areas of bone formation. The cells then develop into chondrocytes that differentiate and become hypertrophic giving rise to osteoblasts which lay down bone matrix resulting in the formation of hydroxyapatite in the matrix. The collagen type-II observed at early culture did not continue throughout the 28 days, instead the cells created a protein dominated ECM that mineralised over culture time. The hMSC nodules at days 21 and 28 each contain two mineral species, that are similar to those observed in native bone. Due to the different mineral factors at days 21 and 28 it is suggested that similar to native bone, the remodeling is continuous throughout the culture and is reflected in a combination of older and newer bone being present. Both the carbonate-to-phosphate ratio and the mineral-to-matrix ratio increased with culture times which further suggests that the various mineral species observed can be compared to native bone. Day $14 \mathrm{hMSC}$ aggregates failed to produce combinations of multiple mineral species and had a significantly lower carbonate-to-phosphate ratio than bone nodules formed at days 21 and 28 of culture. This suggests that although hMSCs at day 14 produced less mineral, the mineral that was detected can be related to the crystalline, non-substituted mineral found in synthetic hydroxyapatite.

One vital issue when considering the transfer of regenerative medicine into the clinical setting is the ability to determine the success of such products to replicate the functionality of their tissue of origin. Here mesenchymal stem cells were obtained from a healthy human donor and were directed towards an osteogenic lineage, samples were taken at seven day intervals from days 7 through to day 28. Differences were observed between hMSCs at early culture and at late culture. hMSCs at days 21 and 28 formed a bone material that had many similarities to that of native bone, namely, increasing carbonate-to-phosphate and mineral-tomatrix ratios and various mineral species. In contrast, hMSCs at day 14 showed various differences with regards to the composition and development. These findings could potentially have a significant impact on the assessment of bone quality and were only possible when combining both biological and materialbased techniques, namely Raman spectroscopy.

\section{Acknowledgements}

The authors would like to acknowledge the support of the Tyndall National Institute. This support was provided through the SFI-funded National Access Programme (Project NAP303). Financial support from the Northern Ireland Department of Employment and Learning (DEL) under the Cross Border Research and Development Funding Programme-Strengthening the All-Island Research Base (Grant Number UU005) is greatly acknowledged.

\section{References}

1 F. R. Rose and R. O. Oreffo, Bone tissue engineering: hope $v$ sype, Biochem. Biophys. Res. Commun., 2002, 292(1), 1-7.

2 I. B. Black and D. Woodbury, Adult rat and human bone marrow stromal stem cells differentiate into neurons, Blood Cells, Mol. Dis., 2001, 27(3), 632-636.

3 D. Bosnakovski, Isolation and multilineage differentiation of bovine bone marrow mesenchymal stem cells, Cell Tissue Res., 2005, 319(2), 243-253.

$4 \mathrm{~S}$. Shirasawa, In vitro chondrogenesis of human synovium-derived mesenchymal stem cells: optimal condition and comparison with bone marrow-derived cells, J. Cell. Biochem., 2006, 97(1), 84-97.

5 S. P. Bruder, et al., Bone regeneration by implantation of purified, culture-expanded human mesenchymal stem cells, J. Orthop. Res., 1998, 16, 155-162.

6 S. P. Bruder, et al., Mesenchymal stem cells in osteobiology and applied bone regeneration, Clin. Orthop. Relat. Res., 1998, S247S256.

7 E. Gentleman, R. J. Swain, N. D. Evans, S. Boonrungsiman, G. Jell, M. D. Ball, T. A. V. Shean, M. L. Oyen, A. Porter and M. M. Stevens, Comparative materials differences revealed in engineered bone as a function of cell-specific differentiation, Nat. Mater., 2009, 8(9), 763-770.

8 S. Stewart, et al., Trends in early mineralization of murine calvarial osteoblastic cultures: a Raman microscopic study, J. Raman Spectrosc., 2002, 33(7), 536-543. 
9 C. P. Tarnowski, M. A. Ignelzi and M. D. Morris, Mineralization of developing mouse calvaria as revealed by Raman microspectroscopy, J. Bone Miner. Res., 2002, 17(6), 1118-1126.

$10 \mathrm{H}$. K. Chiang, et al., In situ Raman spectroscopic monitoring of hydroxyapatite as human mesenchymal stem cells differentiate into osteoblasts, J. Raman Spectrosc., 2009, 40(5), 546-549.

11 I. Notingher and L. L. Hench, Study of osteoblasts mineralisation in-vitro by Raman microspectroscopy, Key Eng. Mater., 2004, 254-256, 769-772.

12 A. Downes, R. Mouras and A. Elfick, Optical spectroscopy for noninvasive monitoring of stem cell differention, J. Biomed. Biotechnol., 2010, 2010, 1-10.

13 C. Rey, et al., Hydroxyl groups in bone mineral, Bone, 1995, 16(5), 583-586.

14 J. Timlin, et al., Spatial distribution of phosphate species in mature and newly generated mammalian bone by hyperspectral Raman imaging, J. Biomed. Opt., 1999, 4(1), 28-34.

15 J. Timlin, A. Carden and M. D. Morris, Chemical microstructure of cortical bone probed by Raman transects, Appl. Spectrosc., 1999, 53 (11), 1429-1435.

16 C. Krafft, et al., Differentiation of individual human mesenchymal stem cells probed by FTIR microscopic imaging, Analyst, 2007, 132, 647-653.

17 R. M. Salasznyk, et al., Focal adhesion kinase signaling pathways regulate the osteogenic differentiation of human mesenchymal stem cells, Exp. Cell Res., 2007, 313(1), 22-37.

18 M. J. Walsh, et al., Fourier transform infrared microspectroscopy identifies symmetric $\mathrm{PO}_{2}^{-}$modifications as a marker of the putative stem cell region of human intestinal crypts, Stem Cells, 2008, 28, 108-118.

19 R. Zhao, L. Quaroni and A. G. Casson, Fourier transform infrared (FTIR) spectromicroscopic characterisation of stem-like cell populations in human esophageal normal and adenocarcinoma cell lines, Analyst, 2010, 135, 53-61.

20 C. Hughes, et al., SR-FTIR spectroscopy of renal epithelial carcinoma side population cells displaying stem cell-like characteristics, Analyst, 2010, 135(12), 3133-3141.

21 M. D. Morris, et al. Early mineralization of normal and pathologic calvaria as revealed by Raman spectroscopy, Proc. SPIE, 2002, 4614(28), 28-39.
22 I. Notingher and L. L. Hench, Raman spectroscopy: potential tool for in-situ characterisation of bone cell differentiation, Key Eng. Mater., 2005, 284-286, 545-548.

23 C. Angela and D. M. Michael, Application of vibrational spectroscopy to the study of mineralized tissues (review), J. Biomed. Opt., 2000, 5(3), 259-268.

24 N. J. Crane, A Nondestructive Method for Monitoring In Vitro Stem Cell Osteogenic Differentiation with Raman Spectroscopic Monitoring, in Raman Spectroscopy Application Note, PerkinElmer, 2009.

25 F. L. Martin, et al., Distinguishing cell types or populations based on the computational analysis of their infrared spectra, Nat. Protoc., 2010, 5(11), 1748-1760.

26 F. Bonnier, et al., Imaging live cells grown on a three dimensional collagen matrix using Raman microspectroscopy, Analyst, 2010, 135 (12), 3169-3177.

27 P. O'Hare, The Role of Inner Pore Topography on Osteoblast Response in Hydroxyapaite Scaffolds, PhD Thesis, 2006.

28 J. E. Aubin, Bone stem cells, J. Cell. Biochem., 1998, 73-82.

29 P. Ducy and G. Karsenty, The family of bone morphogenetic proteins, Kidney Int., 2000, 57, 2207-2214.

30 I. Notingher and L. L. Hench, In situ characterisation of living cells by Raman spectroscopy, Spectroscopy, 2002, 16(2), 43-51.

31 I. Notingher, G. Jell, P. Notingher, I. Bisson, O. Tsigkou, J. M. Polak, M. M. Stevens and L. L. Hench, Multivariate analysis of Raman spectra for in vitro non-invasive studies of living cells, J. Mol. Struct., 2005, 744-747, 179-185.

32 A. L. Boskey and R. Mendelsohn, Infrared spectroscopic characterization of mineralized tissues, Vib. Spectrosc., 2005, 38(12), 107-114.

33 M. Morris, C. Tarnowski and J. Timlin, Multivariate Raman Microscopy: Application to Biological Materials and Synthetic Materials. Microbeam Analysis 2000, Proceedings, 2000, vol. 165, pp. 55-56.

34 G. Penel, et al., MicroRaman spectral study of the $\mathrm{PO}_{4}$ and $\mathrm{CO}_{3}$ vibrational modes in synthetic and biological apatites, Calcif. Tissue Int., 1998, 63(6), 475-481. 